Pacific Journal of Mathematics

INTEGRALLY CLOSED IDEALS AND ASYMPTOTIC PRIME 


\title{
INTEGRALLY CLOSED IDEALS AND ASYMPTOTIC PRIME DIVISORS
}

\author{
L. J. RATLIFF, JR.
}

\begin{abstract}
The first theorem characterizes local (Noetherian) domains that have a height one maximal ideal in their integral closure as those local domains whose maximal ideal $M$ is a prime divisor (= associated prime) of the integral closure $I_{a}$ of all nonzero ideals $I$ contained in large powers of $M$. The second theorem describes (modulo a mild assumption) all local domains $R$ that have the following property: for each ideal $I$ in $R$ and for all large $n$, all the ideals $I^{n}$ and $\left(I^{n}\right)_{a}$ have the same prime divisors.
\end{abstract}

1. Introduction. In [12, (9)], local domains that have a depth one prime divisor of zero in their completion were characterized as those local domains whose maximal ideal $M$ is a prime divisor of all nonzero ideals $I$ contained in large powers of $M$. Also, a similar characterization of a subclass of the class of local domains whose completions have a depth one minimal prime ideal was given in [12, (11)], but $I$ was unable to give an analogous characterization of this entire class in [12]. The first of the main results in this paper, Theorem 1, gives the desired characterization, which was described in the abstruct. This result is actually proved for local rings, and the result [12, (9)] is also extended to this case (in Theorem 0).

As an application of this result, we consider, in $\S 3$, the following class of local domains $R$ : for each ideal $I$ in $R$ and for all large $n$, all the ideals $I^{n}$ and $\left(I^{n}\right)_{a}$ have the same prime divisors. Concerning this class, McAdam and Eakin showed in [4, Prop. 24] that for each ideal $I$ in a Noetherian UFD of altitude two and for all $n \geqq 1$, all the ideals $I^{n}$ and $\left(I^{n}\right)_{a}$ have the same prime divisors. On weakening the conclusion to "for all large $n$," McAdam showed in [5, Thm. 6 and Prop. 8] that this continues to hold for all integrally closed Noetherian domains of altitude two and "integrally closed" is a partly necessary hypothesis. In [13, Prop. 12], $I$ added two other types of local domains that have this latter property, and the second of the main results in this paper, Theorem 4, describes all Noetherian domains that have this property (assuming that integrally closed local domains of altitude three are catenary). The proof of Theorem 4 requires consideration of several cases, and Theorem 1 plays an important role in some of these cases.

Concerning this second class of rings, I am indebted to $\mathrm{S}$. 
McAdam for a stimulating conversation that showed me it was of some interest, and the reader will note his large contribution to the description of the rings in it.

2. Note on the prime divisors of $\left(I^{n}\right)_{a}$. We begin by fixing some notation.

If $R$ is a ring, then $R^{\prime}$ denotes the integral closure of $R$ in its total quotient ring. And if $R$ is local with maximal ideal $M$, then $R^{*}$ denotes the $M$-adic completion of $R$.

For an ideal $I$ in a Noetherian ring $R$, the integral closure of $I$ in $R$ is the set $I_{a}=\{x \in R ; x$ is a root of a polynomial of the form $T^{n}+i_{1} T^{n-1}+\cdots+i_{n}$, where $\left.i_{j} \in I^{j}\right\}$. It is well known that $I_{a}$ is an ideal in $R$ and $I \subseteq I_{a} \subseteq \operatorname{Rad} I$.

We can now prove the first of the main results in this paper. Before this, however, since Theorem 1 is proved in more generality than the analogous result in [12], we first generalize that result to local rings. (Concerning the condition (0): $M=(0)$, it is shown in [15, (3.15.1)] that if $M$ is a prime divisor of zero, then $M$ is a prime divisor of all ideals contained in large powers of $M$.)

THEOREM 0. The following statements are equivalent for a local ring $(R, M)$ such that (0): $M=(0)$ :

(0.1) There exists a depth one prime divisor of zero in $R^{*}$.

(0.2) There exist integers $n$ such that $M$ is a prime divisor of all regular ideals contained in $M^{n}$.

Proof. The proof given in [12] carries over to this more general case, but three minor changes should be made: for $(9.1) \Rightarrow(9.2), I$ is a regular (instead of nonzero) ideal in $R$; and, in the second paragraph of the proof of $(9.2) \Rightarrow(9.1)$ : $a$ should be chosen to be a regular element in $M$ (instead of a nonzero element); and (ii) should be changed to: the intersection of each infinite subset of $\mathscr{P}_{i}$ is a prime divisor of zero.

We now state and prove the first of our main results.

THEOREM 1. The following statements are equivalent for a local ring $(R, M)$ such that altitude $R \geqq 1$ :

(1.1) There exists a depth one minimal prime ideal in $R^{*}$.

(1.2) There exists a positive integer $n$ such that $M$ is a prime divisor of $I_{a}$ for all ideals $I \subseteq M^{n}$ such that height $I \geqq 1$.

Moreover, if $\operatorname{Rad} R=(0)$, then these are also equivalent to:

(1.3) There exists a height one maximal ideal in $R^{\prime}$.

Proof. It was shown in [8, Prop. 3.5] that $(1.1) \Leftrightarrow(1.3)$ when 
$R$ is a local domain, so it readily follows they are equivalent when $\operatorname{Rad} R=(0)$.

Assume (1.1) holds, let $M^{*}=M R^{*}$ be the maximal ideal in $R^{*}$, and let $Z=\operatorname{Rad} R^{*}$. Then it will first be shown that

$$
Z=\bigcap_{n>0}\left(M^{* n}\right)_{a} .
$$

For this, it is clear that $Z \cong\left(M^{* n}\right)_{a}$ for each $n$, so $Z \subseteq \bigcap_{n>0}\left(M^{* n}\right)_{a}$. Therefore $\left[\bigcap_{n>0}\left(M^{* n}\right)_{a}\right] / Z \subseteq \bigcap_{n>0}\left[\left(M^{* n}\right)_{a} / Z\right] \subseteq \bigcap_{n>0}\left[\left(M^{* n}+Z\right) / Z\right]_{a}=$ $\bigcap_{n>0}\left[\left(M^{*} / Z\right)^{n}\right]_{a}$. Now $R^{*} / Z$ is analytically unramified, so $\left(\left(M^{*} / Z\right)^{n}\right)_{a} \subseteq$ $\left(M^{*} / Z\right)^{m(n)}$ with $m(n) \rightarrow \infty$ with $n$, by [16, Lemma 3], so $\bigcap_{n>0}\left[\left(M^{*} / Z\right)^{n}\right]_{a} \subseteq \bigcap_{m>0}\left(M^{*} / Z\right)^{m}=(0)$ in $R^{*} / Z$. Therefore $\left(^{*}\right)$ holds.

Next, since (1.1) holds, let $\bigcap_{1}^{g} q_{i}$ be a normal primary decomposition of the zero ideal in $R^{*}$, where depth $q_{1}=1$ and height $q_{1}=0$. Then, since height $q_{1}=0$, there exists an element $x \in\left(\bigcap_{2}^{g} q_{i}\right)-\operatorname{Rad} q_{1}$, so $x \notin Z$. Therefore, by $\left(^{*}\right)$, there exists an $n$ such that $x \notin\left(M^{* n}\right)_{a}$. Also, since depth $q_{1}=1, \quad x \in \operatorname{Ker}\left(R^{*} \rightarrow R_{P}^{*}\right)$ for all $P \in \operatorname{Spec} R^{*}-$ $\left\{\operatorname{Rad} q_{1}, M^{*}\right\}$, so if $I$ is an ideal in $R$ such that height $I \geqq 1$ and $I: M=I$, then it follows (by considering a primary decomposition of $\left.I R^{*}\right)$ that $x \in I R^{*}$. Therefore if $I \subseteq M^{n}$ then $I_{a} \subseteq\left(M^{n}\right)_{a} \subseteq\left(M^{* n}\right)_{a}$, so $x \notin I_{\alpha} R^{*}$ (by the choice of $n$ ), so it follows that $M$ is a prime divisor of $I_{a}$. Therefore $(1.1) \Rightarrow(1.2)$.

Finally, assume (1.2) holds. Then it continues to hold for $R / Z_{0}$, where $Z_{0}=\operatorname{Rad} R$, since $Z_{0} \subseteq I_{a}$ for all ideals $I$ in $R$. Also, there exists a depth one minimal prime ideal in $R^{*}$ if and only if there exists a depth one minimal prime indeal in $R^{*} / Z_{0} R^{*}=\left(R / Z_{0}\right)^{*}$, so it may be assumed that $\operatorname{Rad} R=(0)$. Let $b$ be a regular element in $M$. Then, by hypothesis, $M$ is a prime divisor of $\left(b^{n} R\right)_{a}$ for all large $n$. Hence, since $\left(b^{n} R\right)_{a}=b^{n} R^{\prime} \cap R$, there exists a (height one) prime divisor $p^{\prime}$ of $b^{n} R^{\prime}$ that lies over $M$. Thus $p^{\prime}$ is a maximal ideal, so $(1.2) \Rightarrow(1.3)$, and $(1.3) \Rightarrow(1.1)$ when $Z_{0}=(0)$, as noted above, so $(1.2) \Rightarrow(1.1)$.

We next give two corollaries of Theorem 1 . The first of these is closely related to two results of Nagata concerning the prime divisors of principal ideals.

CoROllaRy 2. (Cf. [6, (33.11) and (12.6)]). Let B be a Noetherian domain and let $b$ be a nonzero nonunit in $B$. Then the following statements hold:

(2.1) If $P^{\prime} \in \operatorname{Spec} B^{\prime}$ is a prime divisor of $b B^{\prime}$, then $P^{\prime} \cap B$ is a prime divisor of $\left(b^{n} B\right)_{a}$ for all large $n$.

(2.2) If $P \in \operatorname{Spec} B$ is a prime divisor of $\left(b^{n} B\right)_{a}$ for some $n \geqq 1$, then there exists an integer $n$ such that $P$ is a prime divisor of $I_{a}$ 
for all nonzero ideals $I \subseteq P^{(n)}=P^{n} B_{P} \cap B$.

Proof. (2.1) Let $P^{\prime}$ be a prime divisor of $b B^{\prime}$ and let $P=P^{\prime} \cap B$. Then $P^{\prime} B_{(B-P)}^{\prime}$ is a height one maximal ideal in the integral closure $B_{(B-P)}^{\prime}$ of $B_{P}$, so $P B_{P}$ is a prime divisor of $\left(b^{n} B_{P}\right)_{a}$ for all large $n$, by $(1.3) \Rightarrow(1.2)$. But $\left(b^{n} B_{P}\right)_{a}=\left(b^{n} B\right)_{a} B_{P}$, so (2.1) holds.

(2.2) If $P$ is a prime divisor of $\left(b^{n} B\right)_{a}$, then there exists a (height one) prime divisor $P^{\prime}$ of $b^{n} B^{\prime}$ such that $P^{\prime} \cap B=P$. Therefore if $n$ is large and $I$ is a nonzero ideal in $B$ such that $I \subseteq P^{(n)}$, then $P B_{P}$ is a prime divisor of $I_{a} B_{P}=\left(I B_{P}\right)_{a}$, by $(1.3) \Rightarrow(1.2)$, so $(2.2)$ holds.

The next corollary concerns the Rees ring. By definition, the Rees ring of $B$ with respect to an ideal $I$ in $B$ is the graded subring $\mathscr{R}=\mathscr{R}(B, I)=B[t I, u]$ of $B[t, u]$, when $t$ is an indeterminate and $u=1 / t$. It is readily seen that $u^{n} \mathscr{R} \cap B=I^{n}$ and $\left(u^{n} \mathscr{R}\right)_{a} \cap$ $B=\left(I^{n}\right)_{a}$ for all $n \geqq 1$.

It was shown in [11, Thm. 2.5] that $(3.1) \Leftrightarrow(3.2)$, so the main new result in Corollary 3 is that $(3.4) \Rightarrow(3.1)$. However, Rees rings play an important role when considering asymptotic prime divisors, so it was decided to include all four statements in the corollary for ease of reference. Corollary 3 together with [4, Cor. 17] give a useful description of the relationship between the prime divisors of $\left(I^{n}\right)_{a}$ and those of $I^{n}$ for large $n$.

CoRollary 3. Let $I$ be an ideal in a Noetherian domain $B$ and let $\mathscr{R}=\mathscr{R}(B, I)$ be the Rees ring of $B$ with respect to $I$. Then the following statements are equivalent for a prime ideal $P$ in $B$ such that $I \subseteq P$ :

(3.1) $P$ is a prime divisor of $\left(I^{n}\right)_{a}$ for all large $n$.

(3.2) $P$ is a prime divisor of $\left(I^{n}\right)_{a}$ for some $n \geqq 1$.

(3.3) There exists an integer $m$ and a prime divisor $Q$ of $\left(u^{m} \mathscr{R}\right)_{a}$ such that $Q \cap B=P$.

(3.4) There exists a (height one) prime divisor $Q^{\prime}$ of $u \mathscr{R}^{\prime}$ such that $Q^{\prime} \cap B=P$.

Proof. It is clear (3.1) $\Rightarrow(3.2)$. Also, $(3.2) \Rightarrow(3.3)$, since $\left(u^{n} \mathscr{R}\right)_{a} \cap$ $B=\left(I^{n}\right)_{a}$, and $(3.3) \Rightarrow(3.4)$, since $u^{m} \mathscr{R}^{\prime} \cap \mathscr{R}=\left(u^{m} \mathscr{R}\right)_{a}$ and since $u \mathscr{R}^{\prime}$ and $u^{m} \mathscr{R}^{\prime}$ have the same prime divisors.

Finally, if (3.4) holds, then $Q=Q^{\prime} \cap \mathscr{R}$ is a prime divisor of $\left(u^{n} \mathscr{R}\right)_{a}$ for all large $n$, by $(1.3) \Rightarrow(1.2)$. Therefore the proof of [11, Thm. 2.5] shows that $t I \nsubseteq Q$ and $Q \cap B$ is a prime divisor of $\left(I^{n}\right)_{a}$ for all large $n$, so $(3.4) \Rightarrow(3.1)$.

3. On the asymptotic prime divisors of $I^{n}$ and of $\left(I^{n}\right)_{a}$. Before 
stating the main result in this section, we first give three additional definitions

An integral domain $R$ satisfies the altitude formula if for all finitely generated extension domains $A$ of $R$ and all prime ideals $P$ in $A$, height $P+\operatorname{trd}(A / P) /(R / p)=$ height $p+\operatorname{trd} A / R$, where $p=$ $P \cap R$ and $\operatorname{trd} D / C$ denotes the transcendence degree of the integral domain $D$ over its subdomain $C$.

It was shown in [1] that all large powers of an ideal $I$ in a Noetherian ring $R$ have the same prime divisors, and we let $A^{*}(I)$ denote this set, so $A^{*}(I)=\operatorname{Ass}\left(R / I^{n}\right)$ for large $n$. Also, it was shown in [11, Thm. 2.5] that for all large $n$, the ideals $\left(I^{n}\right)_{a}$ have the same prime divisors, and we let $\hat{A}^{*}(I)$ denote this set, so $\hat{A}^{*}(I)=$ Ass $\left(R /\left(I^{n}\right)_{a}\right)$ for large $n$.

It was shown in [11, Thm. 2.5 and Cor. 2.6] that $\hat{A}^{*}(I) \subseteq A^{*}(I)$ always holds. The following result, which is the main result in this section, characterizes (modulo the assumption that all integrally closed local domains of altitude three are catenary) when equality holds for each ideal $I$ in a Noetherian domain. This is a desirable property, since, given: $I$; an integer $k \geqq 1$; and, an ideal $C$ such that $C_{a}=\left(I^{k}\right)_{a}$; then for all ideals $K$ such that $C \cong K \cong\left(I^{k}\right)_{a}$ and for all large $n$, all the ideals $K^{n},\left(K^{n}\right)_{a}, I^{k n}$ and $\left(I k^{n}\right)_{a}$ (and hence also $I^{n}$ and $\left.\left(I^{n}\right)_{a}\right)$ have the same prime divisors. It is therefore somewhat disappointing that the class of such integral domains is rather small.

THEOREM 4. Let be the class of Noetherian domains $B$ such that $\hat{A}^{*}(I)=A^{*}(I)$ for each ideal $I$ in $B$. Then the following statements hold:

(4.1) If altitude $B>3$, then $B \notin \mathscr{A}$.

(4.2) If altitude $B \leqq 3$ and if one of the following statements holds for each maximal ideal $M$ in $B$, then $B \in \mathscr{A}$ :

(4.2.1) Height $M \leqq 1$.

(4.2.2) Height $M=2$ and either $B_{M}=\left(B_{M}\right)^{\prime}$ or there exists a height one maximal ideal in $\left(B_{M}\right)^{\prime}$.

(4.2.3) Height $M=3$, there exists a height one maximal ideal in $\left(B_{M}\right)^{\prime}$, and for all height two prime ideals $P \subset M$ either $B_{P}=\left(B_{P}\right)^{\prime}$ or there exists a height one maximal ideal in $\left(B_{P}\right)^{\prime}$.

(4.3) The only other possible local domains in $\mathscr{A}$ are integrally closed local domains of altitude three that do not satisfy the altitude formula. In fact, assume $(R, M)$ is such an integral domain and let $W$ be the intersection of the depth two minimal prime ideals in $R^{*}$. If $R \in \mathscr{A}$, then each pair of analytically independent elements $b, c$ in $R$ remains analytically independent in $R^{*} / W$. The converse holds if $R / M$ is infinite. 
Before beginning the proof of Theorem 4, the following remarks should be made.

REMARKS. (5.1) It has been an open problem for more than 20 years whether all integrally closed local domains $R$ are catenary. If this holds at least for the case altitude $R=3$, then Theorem 4 describes all Noetherian domains in $\mathscr{A}$ (see Remark 10).*

(5.2) It is shown in [3, Thm. 7 and Remark, p. 728] that there are at most finitely many height two $P \subset M$ for which the condition (in (4.2.3)) "there exists a height one maximal ideal in $\left(B_{P}\right)$ " " can hold.

(5.3) Nagata's examples in [6, Ex. 2, pp. 203-205] in the cases $m=0$ and $r=1$ or 2 are in $\mathscr{A}$, by (4.2.2) and (4.2.3).

(5.4) It is clear that $\mathscr{A}$ is closed under localizations, and using Nagata's example in the case $m=0$ and $r=2$ it can be shown that $\mathscr{A}$ is not closed under passing to factor domains. And, if $(R, M)$ is a local domain such that $R(X)=R[X]_{M R[X]} \in \mathscr{A}$, then $R \in \mathscr{A}$, and the converse in an open problem. (If the converse holds, then (concerning (4.3)) it can be proved that an integrally closed local domain of altitude three that does not satisfy the altitude formula is in $\mathscr{A}$ if and only if the following condition holds: for all pairs of analytically independent elements $b, c$ in $R(X), b, c$ remain analytically independent in $R(X)^{*} / W^{*}$, where $W^{*}$ is the intersection of the depth two minimal prime ideals in $R(X)^{*}$. The proof is quite similar to the proof of Lemma 11.)

(5.5) Lemma 11 proves a somewhat stronger statement than that in (4.3).

Proof of Theorem 4. It clearly sufficient to prove the theorem for the case $B$ is a local domain $R$ and $M$ is its maximal ideal, and it is clear that if height $M=1$, then $R \in \mathscr{A}$.

Now assume height $M=2$. If $R=R^{\prime}$, then $R \in \mathscr{A}$ by [5, Thm. 6]. If $R \neq R^{\prime}$ and $R$ satisfies the altitude formula, then $R \notin \mathscr{A}$ by [5, Prop. 8]. Finally, if $R \neq R^{\prime}$ and if $R$ does not satisfy the altitude formula, then there exists height one maximal ideal in $R^{\prime}$ by [8, Thm. 3.1 and Cor. 3.4(i)]. Let $I$ be a nonzero ideal in $R$. (It is clear that $\hat{A}^{*}((0))=A^{*}((0))$, so it suffices to restrict attention to nonzero ideals.) Then $M \in \widehat{A}^{*}(I)$, by $(1.3) \Longrightarrow(1.2)$, so $M \in A^{*}(I)$, by [11, Thm. 2.5 and Cor. 2.6]. Also, if $P$ is a height one prime ideal in $R$, then clearly $P \in \widehat{A}^{*}(I)$ if and only if $P \in A^{*}(I)$. Therefore $R \in \mathscr{A}$.

Next, assume height $M=3$ and that there exists a height one maximal ideal in $R^{\prime}$. Let $I$ be a nonzero ideal in $R$. Then by $(1.3) \Rightarrow(1.2)$ and [11, Thm. 2.5 and Cor. 2.6], $M \in \hat{A}^{*}(I) \cap A^{*}(I)$. Let 
$P$ be a height two prime ideal in $R$. Then going through the same three cases as in the preceding paragraph, it follows that $P \in \hat{A}^{*}(I)$ if and only if $P \in A^{*}(I)$ when $R_{P}=\left(R_{P}\right)^{\prime}$ and when there exists a height one maximal ideal in $\left(R_{P}\right)^{\prime}$ (so $R_{P} \neq\left(R_{P}\right)^{\prime}$ and $R_{P}$ does not satisfy the altitude formula), and $R_{P} \notin \mathscr{A}$ if $R_{P} \neq\left(R_{P}\right)^{\prime}$ and $R_{P}$ satisfies the altitude formula (and this clearly implies $R \notin \mathscr{A}$ ). From this it readily follows that $R \in \mathscr{A}$ if and only if the conditions of (4.2.3) hold, and this complies the proof of (4.2).

To complete the proof of Theorem 4, it remains to consider the cases: height $M=3$ and there does not exist a height one maximal ideal in $R^{\prime}$; and, height $M \geqq 4$. For these cases, some preliminary information is needed, so the rest of the proof of this theorem is contained in Lemma 6, Corollary 9, Remark 10, and Lemma 11.

Lemma 6 describes a class of altitude three local domains that are not in $\mathscr{A}$.

Lemma 6. Let $(R, M)$ be a local domain such that altitude $R=3$. Assume $R \neq R^{\prime}$ and that there does not exist a height one maximal ideal in $R^{\prime}$. Then $R \notin \mathscr{A}$.

Proof. Since $R \neq R^{\prime}$, let $b \in M$ such that $b R \subset(b R)_{a}$. Let $y \in$ $(b R)_{a}-b R$ and let $I=(b, y M) R$. Then it is shown in [5, Prop. 8] that $M \in A^{*}(I)$, so to complete the proof, it suffices to show that $M \notin \hat{A}^{*}(I)$.

For this, let $\mathscr{R}=R[u, t b]$ be the Rees ring of $R$ with respect to $b R$, let $\mathscr{R}_{1}=R^{\prime}[u, t b]$, and let $\mathscr{S}=R^{\prime}[t b, 1 / t b]$. Then $\mathscr{S}=\mathscr{S}^{\prime}$, since $t b$ is transcendental over $R^{\prime}$, so $\mathscr{R} \subseteq \mathscr{R}_{1} \subseteq \mathscr{R}^{\prime} \subseteq \mathscr{S}=\mathscr{R} 1[1 / t b]$ (since $u=b(1 / t b) \in \mathscr{S}$ ). Suppose there exists a prime divisor $p$ of $u \mathscr{R}^{\prime}$ such that $M=p \cap R$. Then the proof of [11, Thm. 2.5] shows that $t b \notin p$. Hence $p \mathscr{S} \neq \mathscr{S}$, and so $p \mathscr{S} \cap R^{\prime}$ is a maximal ideal (since $p \cap R=M$ ). However, since $t b$ is transcendental over $R^{\prime}$ and height $p=1, p \mathscr{S}=\left(p \mathscr{S} \cap R^{\prime}\right) \mathscr{S}$, hence height $p \mathscr{S} \cap R^{\prime}=1$. But by hypothesis there does not exist a height one maximal ideal in $R^{\prime}$, so no prime divisor of $u \mathscr{R}^{\prime}$ lies over $M$. Therefore $M \notin \hat{A}^{*}(b R)$, by $(3.1) \Rightarrow(3.4)$. Finally, $b R \subseteq I \subseteq(b R)_{a}$, so $\left(I^{n}\right)_{a}=\left(b^{n} R\right)_{a}$ for all $n$, hence $M \notin \hat{A}^{*}(I)$.

It follows from Lemma 6 and the proof of (4.2.3) that the only local domains of altitude three whose inclusion in $\mathscr{A}$ has not been determined are those which are integrally closed. We explicitly consider these rings in Remark 10 and Lemma 11.

The following lemma is of some interest in itself, since it can frequently be used to show that given an ideal $J$ and a prime ideal $P$ such that $J \subseteq P, P \in A^{*}(I)$ for some ideal $I$ between $J$ and $J_{a}$. 
(For example, let $J=K^{[h]}$ in Corollary 8.) The lemma will be used to show that if either altitude $R>3$ or altitude $R=3$ and $R$ satisfies the altitude formula, then $R \notin \mathscr{A}$.

We consider the analytic spread of an ideal $I$ in a local ring in several of the following results. The analytic spread of $I$, denoted $l(I)$, is defined by $l(I)=\operatorname{depth}(M, u) \mathscr{R}(R, I)$. Therefore $l(I) \leqq$ altitude $R$.

Lemma 7. Let $K \subseteq P$ be ideals in a Noetherian ring $B$ such that $P$ is prime, let $h>1$, let $K^{[h]}$ be the ideal generated by the $h$ th powers of the generators of $K$, and let $I=\left(K^{[h]}, P K^{h}\right) B$. If, for infinitely many $n, K^{[h] n} B_{P} \neq K^{h n} B_{P}$, then $P \in A^{*}(I)$. Moreover, if $B_{P}$ is an integral domain that satisfies the altitude formula and height $P>l\left(K B_{P}\right)$, then $P \notin \hat{A}^{*}(I)$.

Proof. It may clearly be assumed that $B$ is local and $P$ is its maximal ideal. Then we have $I^{n} \cong K^{h n}$ and $I^{n}=K^{[h] n}+P K^{h} I^{n-1}$ for all $n$, so $I^{n} \cong K^{[h] n}+P K^{h n}$. Therefore, if $I^{n}=K^{h n}$, then it follows that $K^{h n}=I^{n}=K^{[h] n}$, since $(B, P)$ is local. On the other hand, if $Q \in \operatorname{Spec} B$ is such that $I \leqq Q \subset P$, then clearly $I^{n} B_{Q}=K^{h n} B_{Q}$ for all $n$. Therefore since $I^{n} \subseteq K^{h n}$ it follows (on considering primary decompositions) that if $K^{[h] n} \neq K^{h n}$ for infinitely many $n$, then $P$ must be a prime divisor of $I^{n}$ for infinitely many $n$, hence $P \in A^{*}(I)$, by [1].

If $B_{P}$ is an integral domain that satisfies the altitude formula and height $P>l\left(K B_{P}\right)$, then $P \notin \widehat{A}^{*}(K)$ by [5, Thm. 3]. But clearly $K^{[h]} \cong K^{h} \subseteq\left(K^{[h]}\right)_{a}$, hence $K^{[h]} \subseteq I \subseteq\left(K^{[h]}\right)_{a}$. Therefore $\left(K^{h n}\right)_{a}=$ $\left(K^{[h] n}\right)_{a}=\left(I^{n}\right)_{a}$ for all $n$, so $\hat{A}^{*}(I)=\widehat{A}^{*}(K)$, and so $P \notin \hat{A}^{*}(I)$.

CoROLlary 8. Let $b_{1}, \cdots, b_{g}(g>1)$ be analytically independent elements in a local ring $(R, M)$, let $K=\left(b_{1}, \cdots, b_{g}\right) R$, let $h>1$, and let $I=\left(K^{[h]}, M K^{h}\right) R$, where $K^{[h]}=\left(b_{1}^{h}, b_{2}^{h}, \cdots, b_{g}^{h}\right) R$. Then $M \in$ $A^{*}(I)$. If $R$ is a domain that satisfies the altitude formula and if $g<$ altitude $R$, then $M \notin \hat{A}^{*}(I)$.

Proof. Let $v(J)$ denote the number of elements in a minimal basis of an ideal $J$. Then $v\left(K^{h n}\right)=\left(\begin{array}{c}h n+g-1 \\ g-1\end{array}\right)$ (binomial coefficient) and $v\left(K^{[h] n}\right)=\left(\begin{array}{c}n+g-1 \\ g-1\end{array}\right)$, since $b_{1}, \cdots, b_{g}$ and $b_{1}^{h}, b_{2}^{h}, \cdots, b_{g}^{h}$ are analytically independent in $R$. Therefore $K^{h n} \neq K^{[h] n}$, hence the conclusions follow from Lemma 7 (and the fact that $l(I) \leqq v(I)$ by [7, Lemma 4, p. 151]). 
COROLLARY 9. Let $R$ be a local domain such that either altitude $R=3$ and $R$ satisfies the altitude formula or altitude $R>3$. Then $R \notin \mathscr{A}$.

Proof. Assume first that altitude $R>3$. Then it is shown in [2, Cor. 2.25] that there exist prime ideals $P$ in $R$ such that height $P=$ altitude $R-1$ and $R_{P}$ is unmixed. Fix such a $P$, so height $P \geqq 3$ and $R_{P}$ satisfies the altitude formula, by [8, Thm. 3.1]. Therefore, since the altitude formula is preserved under localization, it suffices to prove that $R \notin \mathscr{A}$ when altitude $R=3$ and $R$ satisfies the altitude formula. For this, let $M$ be the maximal ideal in $R$, let $b, c \in M$ such that height $(b, c) R=2$ and let $I=\left(b^{2}, c^{2}, M b c\right) R$. Then $M \in A^{*}(I)-\hat{A}^{*}(I)$, by Corollary 8 , hence $R \notin \mathscr{A}$.

Concerning Corollary 9, it should be noted that it follows immediately from [5, Thm. 6 and Prop. 8] that a local domain $R$ of altitude $\geqq 2$ that satisfies the altitude formula and is not integrally closed is not in $\mathscr{A}$.

REMARK 10. It is readily seen that if all integrally closed local domains of altitude three are catenary, then all such local domains satisfy the second chain condition for prime ideals, and hence satisfy the altitude formula, by [8, Thm. 3.1]. Therefore if this holds, then $\mathscr{A}$ has been completely determined. If if does not hold, then we have shown (by the proof of (2.4.3), lemma 6, and Corollary 9) that the only other possible local domains in $\mathscr{A}$ are integrally closed, have altitude three, and do not satisfy the altitude formula. We next consider what can be said in this case.

Lemma 11. Let $(R, M)$ be a local domain such that altitude $R=3$ and $R=R^{\prime}$. Assume $R$ does not satisfy the altitude formula and let $W$ be the intersection of the depth two minimal prime ideals in $R^{*}$. Then the following statements hold:

(11.1) If $R \in \mathscr{A}$, then each pair of analytically independent elements in $R$ remains analytically independent in $R^{*} / W$.

(11.2) If $l\left(\left(I R^{*}+W\right) / W\right)=2$ for all ideals $I$ in $R$ such that $l(I)=2$, then $R \in \mathscr{A}$.

(11.3) If $R / M$ is infinite and if each pair of analytically independent elements in $R$ remains analytically independent in $R^{*} / W$, then the hypothesis of (11.2) holds, so the converse of (11.1) also holds.

Proof. Note first that since $R$ does not satisfy the altitude formula, there exists a minimal prime ideal $z$ in $R^{*}$ such that 
depth $z<3$ by [8, Thm. 3.1]. Since $R=R^{\prime}$, depth $z>1$ by $(1.1) \Rightarrow$ (1.3), so $\operatorname{depth} z=2$, and so the ideal $W$ exists.

(11.1) Assume $R \in \mathscr{A}$ and let $b, c$ be analytically independent elements in $R$. Let $I=\left(b^{2}, c^{2}, M b c\right) R$, so $M \in A^{*}(I)$ by Corollary 9, and so $M \in \hat{A}^{*}(I)=\hat{A}^{*}((b, c) R)$, by hypothesis. Therefore by $(3.1) \Rightarrow$ (3.4), there exists a prime divisor $p^{\prime}$ of $u \mathscr{R}^{\prime}$ that lies over $M$, where $\mathscr{R}=R[u, t b, t c]$. Let $N=(M, u) \mathscr{R}$, so $N$ is a depth two prime ideal (by [9, Lemma 4.3] (applied to $R[u]_{(M, u)}$ and $u, b, c$ ) together with [9, Lemma 4.2], since $b, c$ are analytically independent in $R$ ). Now, by the proof of [11, Thm. 2.5], $t b \notin p^{\prime}$ or $t c \notin p^{\prime}$ and $P=p^{\prime} \cap \mathscr{R}$ is not maximal. Therefore, since $N \cong P$, either $\operatorname{depth} P=2$ (so $P=N$ ) or $\operatorname{depth} P=1$. We will first show that $P=N$.

Let $\mathscr{R}^{\sharp}=R^{*}[u, t b, t c]$ and $P^{\sharp}=P \mathscr{R}^{\sharp}$. Then $\mathscr{R}_{P}$ is a dense subspace of $\mathscr{R}_{P}^{\sharp}$, by [9, Lemma 3.2]. Also, there exists a depth one minimal prime ideal in $\left(\mathscr{R}_{P}^{\sharp}\right)^{*}=\left(\mathscr{R}_{P}\right)^{*}$, by $(1.3) \Rightarrow(1.1)$ (since there exists a height one maximal ideal in $\left.\left(\mathscr{R}_{P}\right)^{\prime}\right)$, so there exists a depth one minimal prime ideal in $\mathscr{R}_{P}^{\#}$, since $\mathscr{R}_{P}^{\# \#}$ is pseudo-geometric. Let $w$ be this ideal, so $z=w \cap R^{*}$ is a minimal prime ideal, by [14, Thm. 1.5]. Also, $\mathscr{R}^{\sharp} /\left(w \cap \mathscr{R}^{\sharp}\right) \cong \mathscr{R}\left(R^{*} / z,(b, c) R^{*} / z\right)=$ (say) $\mathscr{R}_{0}$, by [14, Lemma 1.1], and so $P^{\sharp} /\left(w \cap \mathscr{R}^{\sharp}\right)=$ (say) $P_{0}$ is a prime ideal that contains $\left(M^{*} / z, u\right) \mathscr{R}_{0}$. Now $R^{*} / z$ satisfies the altitude formula and height $P^{*} /\left(w \cap \mathscr{R}^{*}\right)=1$, since depth $w=1$, so we have height $P_{0}+$ trd $\left(\mathscr{P}_{0} / P_{0}\right) /\left(\left(R^{*} / z\right) /\left(M^{*} / z\right)\right)=$ height $M^{*} / z+\operatorname{trd} \mathscr{R}_{0} /\left(R^{*} / z\right)$. Thus height $M^{*} / z=\operatorname{trd}\left(\mathscr{R}_{0} / P_{0}\right) /\left(R^{*} / M^{*}\right)=[6,(14.6)]$ altitude $\mathscr{R}_{0} / P_{0}$, so height $M^{*} / z=\operatorname{depth} P_{0} \leqq 2$ (since $\mathscr{R} / P=\mathscr{R}^{\sharp} / P^{\sharp}=\mathscr{R}_{0} / P_{0}$ ). But height $M^{*} / z=\operatorname{depth} z \geqq 2$, so depth $z=$ height $M^{*} / z=\operatorname{depth} P_{0}=2$. Therefore $\operatorname{depth} P=2$, so $P=N$, and so $P_{0}=\left(M^{*} / z, u\right) \cdot \mathscr{R}_{0}$. Let $L=\left(R^{*} / z\right)[u]_{\left(M^{*} / z, u\right)}$. Then $\left(M^{*} / z, u\right) L[t b, t c]$ is a depth two prime ideal, since $P_{0}$ is, so $u, b, c$ are analytically independent in $L$, by [9, Lemma 4.3], and so $b, c$ are analytically independent in $R^{*} / z$. Therefore, since $W \leqq z$ (since depth $z=2$ ) and $b, c$ are analytically independent in $R^{*} / z,[10$, Remark 4.3(i)] shows that $b, c$ are analytically indepedent in $R^{*} / W$.

(11.2) Assume the condition on ideals of analytic spread two holds. Fix a nonzero ideal $I$ in $R$ and let $P \in \operatorname{Spec} R-\{(0), M\}$. Then $R_{P}=\left(R_{P}\right)^{\prime}$, since $R=R^{\prime}$, so $P \in \hat{A}^{*}(I)$ if and only if $P \in A^{*}(I)$ (by [5, Thm. 6] if height $P=2$, and clearly if height $P=1$ ). Therefore it remains to show that $M \in \hat{A}^{*}(I)$ if and only if $M \in A^{*}(I)$.

For this, if $l(I)=1$ then $l(I R(X))=1$. Since $R(X) / M R(X)$ is infinite, there exists an element $b$ in $R(X)$ such that $b R(X) \subseteq$ $I R(X) \subseteq(b R(X))_{a}$, by [7, Cor., p. 151]. Thus, since $R(X)=R(X)^{\prime}$, $(b R(X))_{a}=b R(X)$, hence $I R(X)$ is principal, and so $I$ is principal. Therefore $I^{n}=\left(I^{n}\right)_{a}$ for all $n$, so $\hat{A}^{*}(I)=A^{*}(I)$ and $M \notin A^{*}(I)$. If 
$l(I)=3$, then let $\mathscr{R}=\mathscr{R}(R, I)$. Then depth $(M, u) \mathscr{R}=3$, by hypothesis, so height $(M, u) \mathscr{R}=1$ (since altitude $\mathscr{R}=$ altitude $R+1$ ). Therefore there exists a height one prime ideal $Q$ in $\mathscr{R}$ such that $(M, u) \mathscr{R} \subseteq Q$, so $Q$ is a prime divisor of $u \mathscr{R}$ and of $(u \mathscr{R})_{a}$. Therefore $M=Q \cap R \in \hat{A}^{*}(I)$, by (3.3) $\Rightarrow(3.1)$, so $M \in A^{*}(I)$, by [11, Thm. 2.5 and Cor. 2.6]. Finally, if $l(I)=2$, then the hypothesis implies $l\left(\left(I R^{*}+W\right) / W\right)=2$. Let $\mathscr{R}=\mathscr{R}(R, I), \mathscr{R}^{*}=\mathscr{R}\left(R^{*}, I R^{*}\right), \mathscr{R}_{0}=$ $\mathscr{R}\left(R^{*} / W,\left(I R^{*}+W\right) / W\right)$, and $W^{\sharp}=W R^{*}[t, u] \cap \mathscr{R}^{*}$. Then by the definition of analytic spread, there exists a depth two minimal prime divisor $P_{0}$ of $\left(M^{*} / W, u\right) \mathscr{R}_{0}$. Since $\mathscr{R}_{0} \cong \mathscr{R}^{\sharp} / W^{\sharp}$, by [14, Lemma 1.1], let $P^{\sharp}$ be the pre-image in $\mathscr{R}^{\sharp}$ of $P_{0}$. Then $P^{\#}$ is a depth two minimal prime divisor of $\left(M^{*}, u\right) \mathscr{R}^{\sharp}$. Also, with $P=P^{\sharp} \cap \mathscr{R}, \mathscr{R}_{P}$ is a dense subspace of $\mathscr{R}_{P}^{\#}$, by [8, Lemma 3.2]. Now altitude $\mathscr{R}_{0}=$ altitude $R^{*} / W+1=3$, so depth $P_{0}=2$ implies height $P_{0}=1$. Therefore height $P^{\sharp} / W^{\sharp}=1$, so there exists a depth one minimal prime ideal in $\mathscr{R}_{P}^{\#}$. Therefore there exists a depth one minimal prime ideal in $\left(\mathscr{R}_{P}^{*}\right)^{*}=\left(\mathscr{R}_{P}\right)^{*}$, so there exists a height one minimal ideal, say $N$, in $\left(\mathscr{R}_{P}\right)^{\prime}$, by $(1.1) \Rightarrow(1.3)$. Therefore $M=N \cap R \in \hat{A}^{*}(I)$, by $(3.4) \Rightarrow$ (3.1), and so $M \in A^{*}(I)$, by [11, Thm. 2.5 and Cor. 2.6].

(11.3) Assume $R / M$ is infinite and let $I$ be an ideal in $R$ such that $l(I)=2$. Then, since $R / M$ is infinite, there exist analytically independent elements $b, c$ in $R$ such that $(b, c) R \cong I \subseteq((b, c) R)_{a}$, by [7, Cor. and Thm. 3, p. 151] (applied to $I$ ) together with [7, Thm. 4 , p. 152] (applied to $(b, c) R$ ). By hypothesis, $b, c$ remain analytically independent in $R^{*} / W$, and clearly $(b, c) R^{*} / W \subseteq\left(I R^{*}+W\right) / W \subseteq$ $\left(((b, c) R)_{a} R^{*} /+W\right) / W \subseteq\left((b, c) R^{*} / W\right)_{a}$, so $l\left(\left(I R^{*}+W\right) / W\right) \geqq 2$, by [7, Thm. 2, p. 151]. But altitude $R^{*} / W=2$, so $l\left(\left(I R^{*}+W\right) / W\right) \leqq 2$. Therefore the hypothesis of (11.2) holds, so $R \in \mathscr{A}$, and so the converse of (11.1) holds.

This paper will be closed with the following question and remarks.

QuESTION. If $I$ is an ideal in a local ring such that $l(I) \geqq 2$, then do there exist integers $h$ such that $I^{[h] n} \neq I^{h n}$ for infinitely many $n$ ?

REMARKS. (12.1) It seems to me the answer to the question must certainly be yes, but I have not been able to prove it. If it does hold, then with $R$ as in Lemma 11, $R \in \mathscr{A}$ if and only if the condition in (11.2) holds. For, let $R \in \mathscr{A}$, let $l(I)=2$, let $h$ such that $I^{[h] n} \neq I^{h n}$ for infinitely many $n$, and let $K=\left(I^{[h]}, M I^{h}\right) R$. Then $M \in A^{*}(K)$, by Lemma 8 , so $M \in \hat{A}^{*}(K)$ since $R \in \mathscr{A}$. Therefore $M \in \hat{A}^{*}\left(I^{h}\right)=\hat{A}^{*}(I)$. Thus essentially as in the proof of (11.1) (but 
using the definition of $l(I)$ to get a depth two prime divisor of $(M, u) \mathscr{R}(R, I)$ rather than analytical independence), it follows that $\operatorname{depth}\left(M^{*} / W, u\right) \mathscr{R}\left(R^{*} / W,\left(I R^{*}+W\right) / W\right)=2$, so $l\left(\left(I R^{*}+W\right) / W\right)=2$.

(12.2) Actually, for the result in (12.1) to hold, it would be sufficient for the following to hold: if $l(I)=2$, then there exist integers $g$ and $h$ and ideals $C$ and $K$ such that $C_{a}=\left(I^{g}\right)_{a}, C \leqq K \leqq$ $\left(I^{g}\right)_{a}$, and $K^{[h] n} \neq K^{h n}$ for infinitely many $n$. (For then, by Lemma 8, $M \in A^{*}\left(K^{*}\right)$, where $K^{*}=\left(K^{[h]}, M K^{h}\right) R$, so $M \in \widehat{A}^{*}\left(K^{*}\right)$, since $R \in \mathscr{A}$, and so $M \in \hat{A}^{*}\left(K^{h}\right)=\hat{A}^{*}\left(I^{g h}\right)=\hat{A}^{*}(I)$.)

Added in proof. It was recently shown by T. Ogoma in Noncatenary pseudo-geometric normal rings, Japan J. Math., (to appear), that there exist integrally closed local domains of altitude three which are not catenary.

\section{REFERENCES}

1. M. Brodmann, Asymptotic stability of Ass $\left(R / I^{n}\right)$, Proc. Amer. Math. Soc., 74 (1979), 16-18.

2. M. Hochster and L.J. Ratliff, Jr., Five theorems on Macaulay rings, Pacific J. Math., 44 (1973), 147-172.

3. S. McAdam, Saturated chains in Noetherian rings, Indiana Univ. Math. J., 23 (1974), 719-728.

4. S. McAdam and P. Eakin, The asymptotic Ass, J. Algebra, 61 (1979), 71-81.

5. S. McAdam, Asymptotic prime divisors and analytic spreads, Proc. Amer. Math. Soc., 80 (1980), 555-559.

6. M. Nagata, Local Rings, Interscience Tracts 13, Interscience, New York, 1962.

7. D. G. Northcott and D. Rees, Reductions of ideals in local rings, Proc. Cambridge Philos. Soc., 50 (1954), 145-158.

8. L.J. Ratliff, Jr., On quasi-unmixed local domains, the altitude formula, and the chain condition for prime ideals (I), Amer. J. Math., 91 (1969), 508-528.

9. - On quasi-unmixed local domains, the altitude formula, and the chain condition for prime ideals (II), Amer. J. Math., 92 (1970), 99-144.

10. - Characterizations of catenary rings, Amer. J. Math., 92 (1971), 1070-1108.

11. _- On prime divisors of $I^{n}, n$ large, Michigan Math. J., 23 (1976), 337-352.

12. - Two theorems on the prime divisors of zero in completions of local domains, Pacific J. Math., 81 (1979), 537-545.

13. - Note on asymptotic prime divisors, analytic spreads, and the altitude formula, Proc. Amer. Math. Soc., (to appear).

14. D. Rees, $A$ note on form rings and ideals, Mathematika, 4 (1957), 51-60.

15. D. Rush and L. J. Ratliff, Jr., Notes on ideal covers and associated primes, Pacific J. Math., 73 (1977), 169-191.

16. M. Sakuma and H. Okuyama, On a criterion for analytically unramification of a local ring, J. Gakugei Tokushima Univ., 15 (1966), 36-38.

Received August 29, 1979. Research on this paper was supported in part by the National Science Foundation, Grant MCS 77-00951-A02.

UNIVERSITY OF CALIFORNIA

RIVERSIDE, CA 92521 


\section{PACIFIC JOURNAL OF MATHEMATICS}

\section{EDITORS}

DONALD BABBITT (Managing Editor)

University of California

Los Angeles, CA 90024

Hugo RossI

University of Utah

Salt Lake City, UT 84112

C. C. MOORE and ANDREW OGG

University of California

Berkeley, CA 94720

\section{J. DugundjI}

Department of Mathematics

University of Southern California

Los Angeles, CA 90007

R. FinN and J. Milgram

Stanford University

Stanford, CA 94305

\section{ASSOCIATE EDITORS}
E. F. BeCKENBACH
B. H. Neumann
F. WoLF
K. YoSHIDA

\section{SUPPORTING INSTITUTIONS}

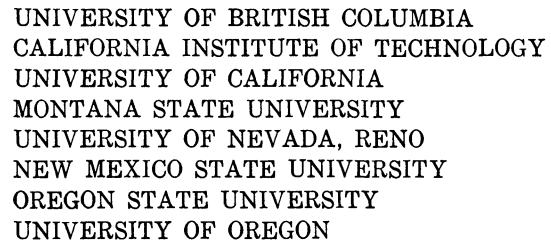

UNIVERSITY OF BRITISH COLUMBIA CALIFORNIA INSTITUTE OF TECHNOLOGY UNIVERSITY OF CALIFORNIA MONTANA STATE UNIVERSITY UNIVERSITY OF NEVADA, RENO NEW MEXICO STATE UNIVERSITY OREGON STATE UNIVERSITY UNIVERSITY OF OREGON

\author{
UNIVERSITY OF SOUTHERN CALIFORNIA \\ STANFORD UNIVERSITY \\ UNIVERSITY OF HAWAII \\ UNIVERSITY OF TOKYO \\ UNIVERSITY OF UTAH \\ WASHINGTON STATE UNIVERSITY \\ UNIVERSITY OF WASHINGTON
}

The Supporting Institutions listed above contribute to the cost of publication of this Journal, but they are not owners or publishers and have no responsibility for its content or policies.

Mathematical papers intended for publication in the Pacific Journal of Mathematics should be in typed form or offset-reproduced, (not dittoed), double spaced with large margins. Please do not use built up fractions in the text of the manuscript. However, you may use them in the displayed equations. Underline Greek letters in red, German in green, and script in blue. The first paragraph or two must be capable of being used separately as a synopsis of the entire paper. Please propose a heading for the odd numbered pages of less than 35 characters. Manuscripts, in triplicate, may be sent to any one of the editors. Please classify according to the scheme of Math. Reviews, Index to Vol. 39. Supply name and address of author to whom proofs should be sent. All other communications should be addressed to the managing editor, or Elaine Barth, University of California, Los Angeles, California, 90024.

50 reprints to each author are provided free for each article, only if page charges have been substantially paid. Additional copies may be obtained at cost in multiples of 50 .

The Pacific Journal of Mathematics is issued monthly as of January 1966. Regular subscription rate: $\$ 84.00$ a year (6 Vols., 12 issues). Special rate: $\$ 42.00$ a year to individual members of supporting institutions.

Subscriptions, orders for numbers issued in the last three calendar years, and changes of address shoud be sent to Pacific Journal of Mathematics, P.O. Box 969, Carmel Valley, CA 93924, U.S.A Old back numbers obtainable from Kraus Periodicals Co., Route 100, Millwood, NY 10546.

PUBLISHED BY PACIFIC JOURNAL OF MATHEMATICS, A NON-PROFIT CORPORATION

Printed at Kokusai Bunken Insatsusha (International Academic Printing Co., Ltd.). 8-8, 3-chome, Takadanobaba, Shinjuku-ku, Tokyo 160, Japan. 


\section{Pacific Journal of Mathematics}

\section{Vol. 91, No. 2 December, 1980}

Victor P. Camillo and Julius Martin Zelmanowitz, Dimension modules ... . . 249

Yonina S. Cooper, Stable sequences in pre-abelian categories ........... 263

Chandrakant Mahadeorao Deo and H. Ship-Fah Wong, On Berry-Esseen approximation and a functional LIL for a class of dependent random fields.........................................

H. P. Dikshit and S. N. Dubey, $|C, 1|$ summability of series associated with

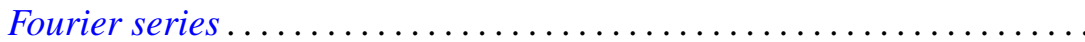

M. Edelstein, On the homomorphic and isomorphic embeddings of a semiflow into a radial flow.

Gilles Godefroy, Compacts de Rosenthal ..................... 293

James Guyker, Commuting hyponormal operators ................ 307

Thomas Eric Hall and Peter R. Jones, On the lattice of varieties of bands of

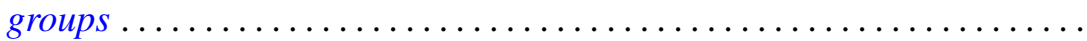

Taqdir Husain and Saleem H. Watson, Topological algebras with orthogonal Schauder bases ....................................

V. K. Jain, Some expansions involving basic hypergeometric functions of two variables. . .

Joe W. Jenkins, On group actions with nonzero fixed points ........... 363

Michael Ellsworth Mays, Groups of square-free order are scarce ........ 373

Michael John McAsey, Canonical models for invariant subspaces... 377

Peter A. McCoy, Singularities of solutions to linear second order elliptic partial differential equations with analytic coefficients by approximation methods...

Terrence Millar, Homogeneous models and decidability.

Stephen Carl Milne, A multiple series transformation of the very well poised

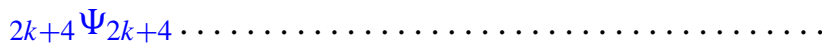

Robert Olin and James E. Thomson, Irreducible operators whose spectra are spectral sets...

Robert John Piacenza, Cohomology of diagrams and equivariant singular

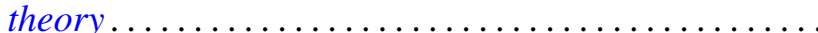

Louis Jackson Ratliff, Jr., Integrally closed ideals and asymptotic prime divisors

Robert Breckenridge Warfield, Jr., Cancellation of modules and groups and stable range of endomorphism rings.................

B. J. Day, Correction to: "Locale geometry" ...............

Stanley Stephen Page, Correction to: "Regular FPF rings" ... 